\title{
Thermal Equilibrium and Density Limit in Tokamak-Reactor
}

\author{
D.Kh. Morozov ${ }^{1,2, *}$, S.N. Vafin $^{1}$, A.A. Mavrin ${ }^{1}$ \\ ${ }^{1}$ Plasma Physics Department, National Research Centre "Kurchatov Institute", 123182, Moscow, Russia \\ ${ }^{2}$ Chair of Plasma Physics, National Research Nuclear University MEPhI, 115409, Moscow, Russia \\ ${ }^{*}$ Corresponding Author: dmitry.morozov.41@mail.ru
}

\begin{abstract}
The problem of the thermal equilibrium and density limit in tokamak-reactor are analyzed. The empirical Greenwald criterion determining the critical plasma density may be exceeded in tokamaks with auxiliary heating. One may expect that the fusion power may increase the critical plasma density. The thermal balance in tokamak-reactors is discussed. The critical density is defined by the equality of heating (auxiliary plus fusion) power and radiation losses at the plasma edge. The influence of fusion power input as well as auxiliary heating on the critical density is studied. The analytic model is based on some simplifying assumptions. The auxiliary heating as well as the thermonuclear one is assumed to be localized at the center of the plasma column. The slab geometry is assumed for simplicity. It is shown that the critical density rises up increasing the fusion output drastically using the feedback control.
\end{abstract}

Keywords Tokamak-Reactor, Thermal Equilibrium, Density Limit, Impurity, Feedback Control

\section{Introduction}

The problems of thermal equilibrium and density limit in contemporary tokamaks have been discussed by many authors. As it is well known a part of disruptions in contemporary tokamaks are related to the radiation collapse [1,2]. The density limit related to the total re-radiation of $\alpha$ - particle power in tokamak reactor has been discussed in [3]. However the authors of [3] didn't take into account auxiliary heating and also ignored the thermal instability produced by fusion power. Experimental results show that the thermal quench takes place if the radiated power for light impurities achieves approximately 90\% [4]. For simplicity we will suppose that the total power is radiated by impurities. The empirical Greenwald's criterion [5] $n_{\mathrm{c}} \sim I$ determining the critical plasma density may be exceeded in tokamaks with auxiliary heating. Here $I$ is the total toroidal current in plasma. As it is shown in experiments an auxiliary heating increases the value of $n_{\mathrm{c}}$ by factor 1.5 and more [1,6-8]. One may expect that the fusion power also can increase the critical plasma density. Such problem for a tokamak-reactor is analyzed in the present paper. As it is shown below the thermal equilib- rium is possible for very high densities. However the thermal equilibrium for extremely high density values is unstable [9], which can be stabilized by the feedback control. The auxiliary heating power at such regimes may be small. The ratio of the fusion power to the auxiliary may be respectively very high and the thermal loading at the divertor plate may be small.

In order to describe the regime mentioned above the qualitative model is considered, in which the slab geometry is used. The critical density is defined by the equality of the heating power (auxiliary plus fusion) and the radiation losses at the plasma edge. The influence of fusion power input as well as auxiliary heating on the critical density is studied. Also the overheating thermal instability and its suppression by feedback control are analyzed.

The paper is organized as following. The thermal equilibrium and stability for plasma density close to the critical one is discussed in Section 2. The qualitative model is investigated analytically. The thermal quench and the thermal instability leading to the ignition are discussed. Results are discussed in Conclusion.

\section{Thermal balance}

The thermal equilibrium is described by the equation

$$
3 n \frac{\partial T}{\partial t}=\nabla \kappa_{\perp} \nabla T+P_{\alpha}-Q_{\mathrm{rad}}
$$

Here $\kappa_{\perp}$ is the thermal conductivity, $P_{\alpha}$ is the fusion power input produced by $\alpha$ - particles, and $Q$ describes the radiation losses. The latest ones are proportional to the background plasma density and the impurity one,

$$
Q_{\mathrm{rad}}=n n_{\mathrm{I}} L(T) .
$$

Here $L(T)$ is the function of the temperature only. As it is shown in [10] the significantly two dimensional transport may be modeled by one dimensional equation. The boundary condition at the plasma edge takes the form $T(a+\Delta)=0$, where $\Delta$ is the SOL thickness. The value $\Delta$ is small in comparison to the minor radius, and one can put $T(a)=0$. The condition mentioned above is acceptable for L-regime. At the H-regime the temperature pedestal is located near the separatrix. The thermal flux is continuous. The temperature 
of the zone behind the pedestal may be expressed in terms of $T=T_{1}+\delta T$, where $\delta T$ is the temperature jump at the pedestal. Hence, one can use the modified temperature,

$$
T=\left\{\begin{array}{c}
T, \text { if } r<r_{\mathrm{p}} \\
T_{1}, \text { if } r>r_{\mathrm{p}} .
\end{array}\right.
$$

Here $r_{\mathrm{p}}$ is the distance from the pedestal to the center. Usually the value $\delta T$ is significantly smaller than the central temperature.

Also, the description is acceptable for so called Radiatively Improved (RI) mode [11].

The steady-state analytic model is based on some simplifying assumptions. The slab geometry is assumed. The auxiliary heating may be supposed to be localized at the plasma center. The boundary condition at the center takes the form

$$
-\left.\kappa_{\perp} \frac{\mathrm{d} T}{\mathrm{~d} r}\right|_{r=0}=M
$$

One can put approximately for the temperature interval $2 \mathrm{keV}$ $<T<10 \mathrm{keV}$

$$
P_{\alpha}=\frac{n^{2}}{4}\langle\sigma v\rangle E_{\alpha} \approx A T^{2} n^{2}
$$

Here $A \approx 0.88 \cdot 10^{-24} \mathrm{~m}^{3} \mathrm{~s}^{-1} \mathrm{eV}^{-1}$ is the normalizing factor. $E_{\alpha}=3.52 \mathrm{MeV}$ is the energy of the alpha particle. Sign $<>$ means the averaging of the value $\sigma v$ over velocities. Concentrations of Deuterium and Tritium are equal, $n_{\mathrm{D}}=n_{\mathrm{T}}=n / 2$. The plasma density is supposed to be independent on $r$, and the impurity density is proportional to the plasma density, $n_{\mathrm{I}}=\lambda n$.

The equation (1) may be integrated for the steady-state regime. Taking into account $\mathrm{d} T / \mathrm{d} r \leq 0$, one can get

$$
\begin{gathered}
\kappa_{\perp} \frac{\mathrm{d} T}{\mathrm{~d} r} \cdot \frac{\mathrm{d}}{\mathrm{d} r}\left(\kappa_{\perp} \frac{\mathrm{d} T}{\mathrm{~d} r}\right)=\kappa_{\perp} \frac{\mathrm{d} T}{\mathrm{~d} r} \cdot\left(Q_{\mathrm{rad}}-P_{\alpha}\right) \\
\left.\left(\kappa_{\perp} \frac{\mathrm{d} T}{\mathrm{~d} r}\right)^{2}\right|_{r}-\left.\left(\kappa_{\perp} \frac{\mathrm{d} T}{\mathrm{~d} r}\right)^{2}\right|_{0} ^{T}=2 \int_{T_{0}}^{T}\left(n n_{\mathrm{I}} L(T)-P_{\alpha}\right) \kappa_{\perp} \mathrm{d} T .
\end{gathered}
$$

Here $T_{0}$ is the temperature at the center. Using a boundary condition (3) one can get

$$
\begin{gathered}
\frac{\mathrm{d} T}{\mathrm{~d} r}=-\frac{1}{\kappa_{\perp}}\left(M^{2}-2 \int_{T}^{T_{0}}\left(n n_{\mathrm{I}} L(T)-P_{\alpha}\right) \kappa_{\perp} \mathrm{d} T\right)^{1 / 2} \\
\int_{T}^{T_{0}} \frac{\kappa_{\perp} \mathrm{d} T}{\left(M^{2}-2 \int_{T}^{T_{0}}\left(n n_{\mathrm{I}} L(T)-P_{\alpha}\right) \kappa_{\perp} \mathrm{d} T\right)^{1 / 2}}=r .
\end{gathered}
$$

The critical density is achieved if the total power input is radiated inside the vacuum vessel including the divertor volume, i.e. the thermal flux through the plasma boundary is equal to zero [1]. Integrating equation (5) over the plasma volume one can get for the critical regime

$$
M^{2}=2 \int_{0}^{T_{0}}\left(n n_{\mathrm{I}} L(T)-P_{\alpha}\right) \kappa_{\perp} \mathrm{d} T .
$$

It means that the total power produced by the auxiliary heating and fusion powers is equal to the radiation losses.

The equation (7) yields

$$
\int_{0}^{T_{0}} \frac{\kappa_{\perp} \mathrm{d} T}{\left(M^{2}-2 \int_{T}^{T_{0}}\left(n n_{\mathrm{I}} L(T)-P_{\alpha}\right) \kappa_{\perp} \mathrm{d} T\right)^{1 / 2}}=a .
$$

Here $a$ is the minor tokamak radius. The expression for the denominator in (9) may be simplified as

$$
\begin{gathered}
F^{2}(T) \equiv M^{2}-2 \int_{T}^{T_{0}}\left(n n_{\mathrm{I}} L-P_{\alpha}\right) \kappa_{\perp} \mathrm{d} T= \\
=M^{2}-2 \int_{0}^{T_{0}}\left(n n_{\mathrm{I}} L-P_{\alpha}\right) \kappa_{\perp} \mathrm{d} T+2 \int_{0}^{T}\left(n n_{\mathrm{I}} L-P_{\alpha}\right) \kappa_{\perp} \mathrm{d} T= \\
=2 \int_{0}^{T}\left(n n_{\mathrm{I}} L-P_{\alpha}\right) \kappa_{\perp} \mathrm{d} T .
\end{gathered}
$$

Finally, one can find the set of two equations determining the values $n$ and $T_{0}$ :

$$
\left\{\begin{array}{l}
M^{2}=2 \int_{0}^{T_{0}}\left(n n_{\mathrm{I}} L(T)-P_{\alpha}\right) \kappa_{\perp} \mathrm{d} T \\
\int_{0}^{T_{0}}\left(2 \int_{0}^{T}\left(n n_{\mathrm{I}} L(T)-P_{\alpha}\right) \kappa_{\perp} \mathrm{d} T\right)^{-1 / 2} \kappa_{\perp} \mathrm{d} T=a .
\end{array}\right.
$$

It is well known from modeling and experiments [12] that the thermal conductivity increases with the minor radius. For simplicity the heat conductivity may be represented by the step function,

$$
\kappa_{\perp}=\left\{\begin{aligned}
\kappa_{0}, & 0 \leq r<r_{*} \\
\mu \kappa_{0}, & r_{*}<r \leq a, \mu>1 .
\end{aligned}\right.
$$

The value $r_{*}$ is chosen as $0.9 a$. The function $F(T)$ may be separated into two parts. The first part for small (less than two hundreds eV) practically doesn't depend on the fusion power input because the latest is extremely small. For large values of $T$ the function $g \equiv \int_{0}^{T} L(T) \mathrm{d} T$ is practically constant (see Fig. 1).

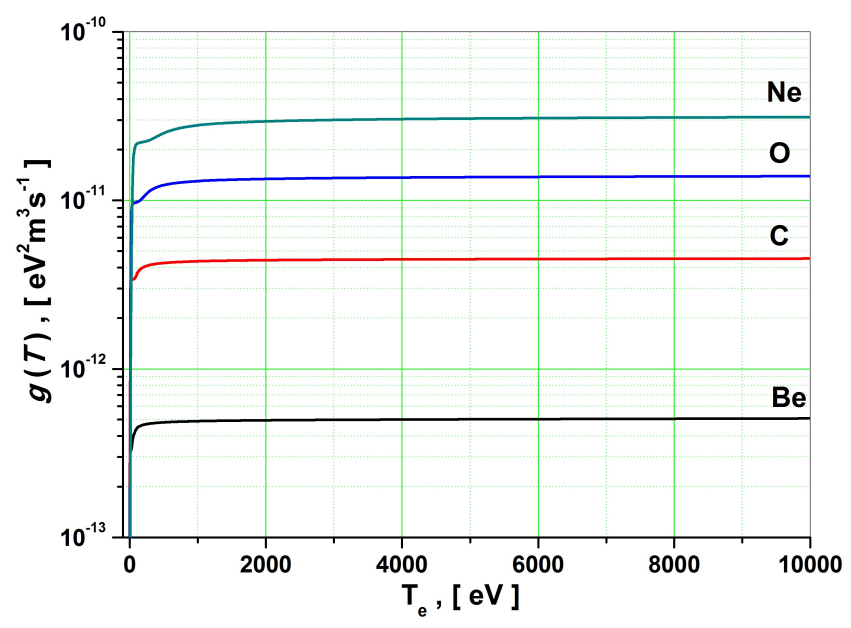

Figure 1. The function $g$ for typical impurities: Beryllium, Carbon, Oxygen, Neon.

Hence, one can write approximately

$$
F \approx \sqrt{\kappa_{0}\left(\mu n n_{\mathrm{I}} g-\int_{0}^{T} P_{\alpha} \mathrm{d} T\right)},
$$

where $g$ depends on the impurity atomic number. For light impurities the bremsstrahlung may be ignored. Using the results of [13-14] one can calculate $g$ for typical impurities (see Table 1). 
Table 1. The integral $g$ for typical impurities: Beryllium, Carbon, Oxygen, Neon.

\begin{tabular}{cc}
\hline Impurity & $g\left[\mathrm{eV}^{2} \mathrm{~m}^{3} \mathrm{~s}^{-1}\right]$ \\
\hline Beryllium & $5.0 \cdot 10^{-13}$ \\
Carbon & $4.5 \cdot 10^{-12}$ \\
Oxygen & $1.4 \cdot 10^{-11}$ \\
Neon & $3.0 \cdot 10^{-11}$ \\
\hline
\end{tabular}

The relatively cold plasma with high heat conductivity is localized in the narrow region. Hence, the heat conductivity in the nominator of (9) may be replaced by $\kappa_{0}$. Using the expression (4) one can find

$$
\int_{0}^{x_{0}} \frac{\mathrm{d} x}{\sqrt{1-x^{3}}}=a n \sqrt{\frac{2 \lambda \mu g}{\kappa_{0}}}\left(\frac{A}{3 \lambda \mu g}\right)^{1 / 3} .
$$

Here $x_{0}=T_{0}\left(\frac{A}{3 \lambda \mu g}\right)^{1 / 3}$. The left hand side of (14) $G\left(x_{0}\right) \equiv$ $\int_{0}^{x_{0}} \frac{\mathrm{d} x}{\sqrt{1-x^{3}}}$ is calculated numerically (Fig. 2).

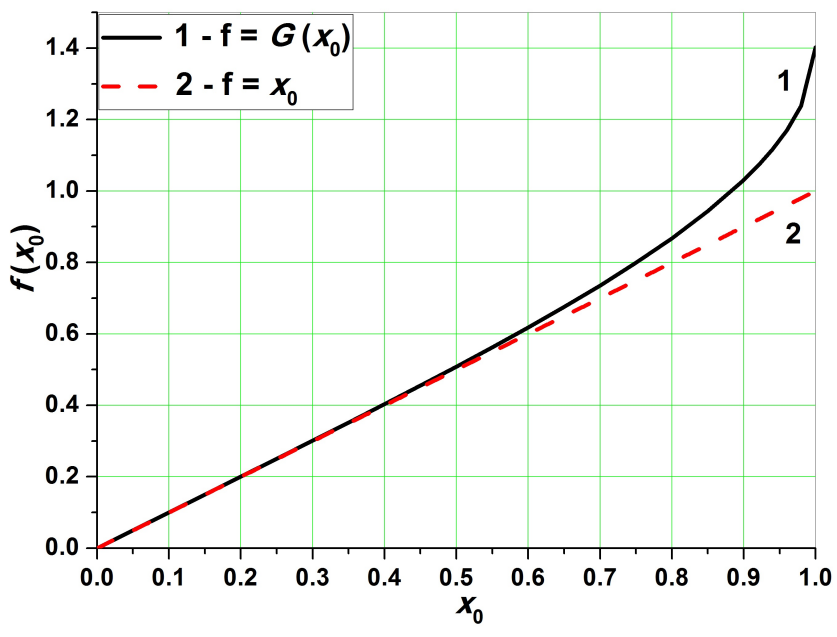

Figure 2. The linear and $G\left(x_{0}\right)$ functions.

At least for $x_{0}<0.75$ the function $G\left(x_{0}\right)$ may be approximately equal to the linear function $G\left(x_{0}\right) \approx x_{0}$. The equation (11.2) yields

$$
T_{0}=a n \sqrt{\frac{\lambda \mu g}{\kappa_{0}}} .
$$

Substituting (15) into (11.1) one can get

$$
\lambda \mu g \cdot n^{2}-\frac{a^{3} A}{3}\left(\frac{2 \lambda \mu g}{\kappa_{0}}\right)^{3 / 2} \cdot n^{5}=\frac{M^{2}}{2 \kappa_{0}} .
$$

The numerical solution of the equations (15-16) is shown in Fig. 3A. The left hand side of (16) is represented by the curve having the maximum. The right hand side is represented by the horizontal straight line. Figures $3 \mathrm{~B}$ and $3 \mathrm{C}$ show the plasma density $n$ and the central temperature $T_{0}$ dependence on the total auxiliary heating power $W_{\text {aux }}=M \cdot 2 \pi R \cdot 2 \pi a$.

The left hand side of (16) has a maximum. The rising part of the curve (solid line) separates the stable region of regimes from the unstable one. At the stable region the fusion impact is small. The total heating exceeds radiation losses, and it is compensated by the heat conductivity. At the unstable region (dashed line) the radiation losses exceed the total heating, and the thermal quench occurs. The dashed line separates two unstable regions. The fusion impact is significant.
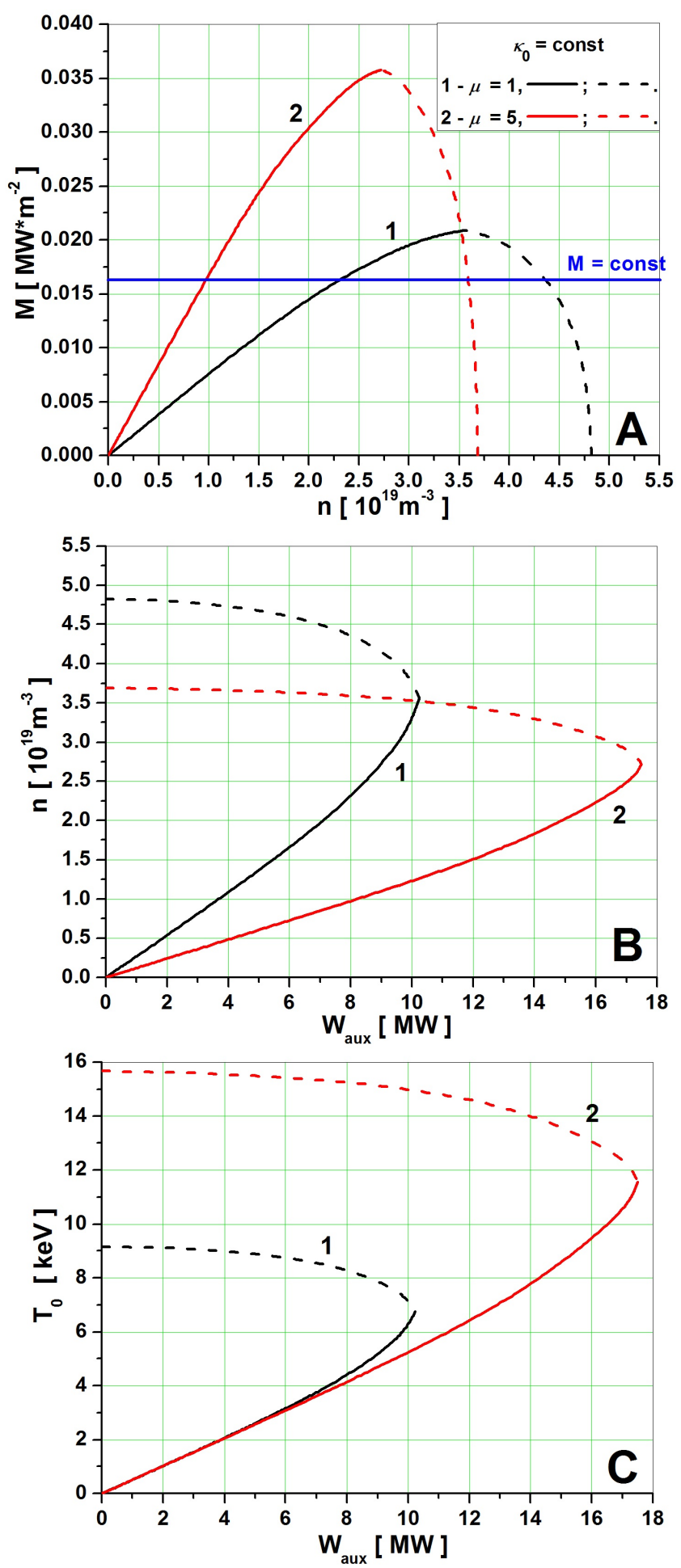

Figure 3. The numerical solution of the equations (15-16) for model $\kappa_{0}=$ const. Here impurity is Carbon, $g=4.5 \cdot 10^{-12} \mathrm{eV}^{2} \mathrm{~m}^{3} \mathrm{~s}^{-1}$. Heat conductivity $\kappa_{0}=5 \cdot 10^{19} \mathrm{~m}^{-1} \mathrm{~s}^{-1}$. Impurity relative concentration $\lambda=0.05$; the minor radius $a=2 \mathrm{~m}$ and the major radius $R=6.2 \mathrm{~m}$.

It rises strongly with the temperature and density. If the auxiliary heating is small the thermal quench takes a place. If the auxiliary heating is high enough the overheating instability [9] develops. Hence, the discharge moves to the ignition. However the regime related to the critical density may be stabilized by feedback control. Hence the discharge can be realizes as the controlled regime with very small level of the auxiliary heating and extremely large relation of the fusion power to the auxiliary heating power.

The maximal value of the left hand side (16) is defined by 
the expression

$$
M_{\max }^{2}=\frac{3}{5} \frac{\kappa_{0}^{2}}{a^{2}}\left(\frac{6 \lambda \mu g}{5 A}\right)^{2 / 3} .
$$

It is achieved with

$$
n=n_{*}=\frac{\sqrt{\kappa_{0}}}{a}\left(\frac{3}{5 A \sqrt{2 \lambda \mu g}}\right)^{1 / 3}
$$

For simplicity it was supposed above, $\kappa_{0}=$ const.

For the scaling $\chi_{0}=$ const it is easy to find

$$
\begin{gathered}
T_{0}=a \sqrt{\frac{\lambda \mu g n}{\chi_{0}}} ; \\
n^{3}\left(\lambda \mu g-\frac{a^{3} A}{3}\left(\frac{2 \lambda \mu g}{\chi_{0}}\right)^{3 / 2} \cdot n^{3 / 2}\right)=\frac{M^{2}}{2 \chi_{0}} ; \\
M_{\max }^{2}=\frac{1}{3}\left(\frac{\chi_{0}}{a^{2}}\right)^{4} \frac{a^{2}}{A^{2}} ; \\
n_{*}=\frac{\chi_{0}}{a^{2}}\left(2 \lambda \mu g A^{2}\right)^{-1 / 3} .
\end{gathered}
$$

The numerical solution of the equations (19-20) is shown in Fig. 4. One can see that the expressions (17) and (21) define the minimal auxiliary heating power which provides the transition to the ignition trough the thermal instability. It is interesting to note that for this case $n_{*}=\frac{1}{\tau_{E}}\left(2 \lambda \mu g A^{2}\right)^{-1 / 3}$, and the maximal auxiliary heating power does not depend on the impurity atomic number.

The minimal minor radius is defined by the equation (17), i.e.

$$
a_{\min }=\sqrt{\frac{3}{5}} \frac{\kappa_{0}}{M_{\max }}\left(\frac{6 \lambda \mu g}{5 A}\right)^{1 / 3} .
$$

The value of $a_{\min }$ is restricted by the maximal power of heating sources. Contemporary source power doesn't exceed the value $M_{\max }=0.05 \mathrm{MWm}^{-2}$ (ITER project). Hence, the minimal minor radius may be estimated as $a_{\text {min }} \approx 0.8 \mathrm{~m}$. Therefore, the tokamak-reactor may be a machine of JET like sizes.

One can see that the expression (17) defines the minimal auxiliary heating power which provides the transition to the ignition trough the thermal instability. In this case the auxiliary heating power is a strong function of minor radius. Hence, the minimal tokamak size is limited by maximal auxiliary heating sources.

\section{Conclusions}

The problem of the fusion energy maximization in tokamaks is discussed. The discharge disruption is supposed to be initiated by the total radiation of the power input including fusion power in $\alpha$ - particle as well as the auxiliary heating. Other reasons of the disruption like MHD instabilities are not discussed. The critical auxiliary power as a function of a critical density is obtained. Such dependence has a maximum. If the density exceeds the critical one (solid line in Fig. 3A) near the rising branch the thermal quench occurs. If the density exceeds the critical one near the decreasing branch (dashed line in Fig. 3A) the overheating thermal instability develops. However the overheating instability may be stabilized by feedback control. The controlled fusion reaction may be achieved for large densities and small auxiliary
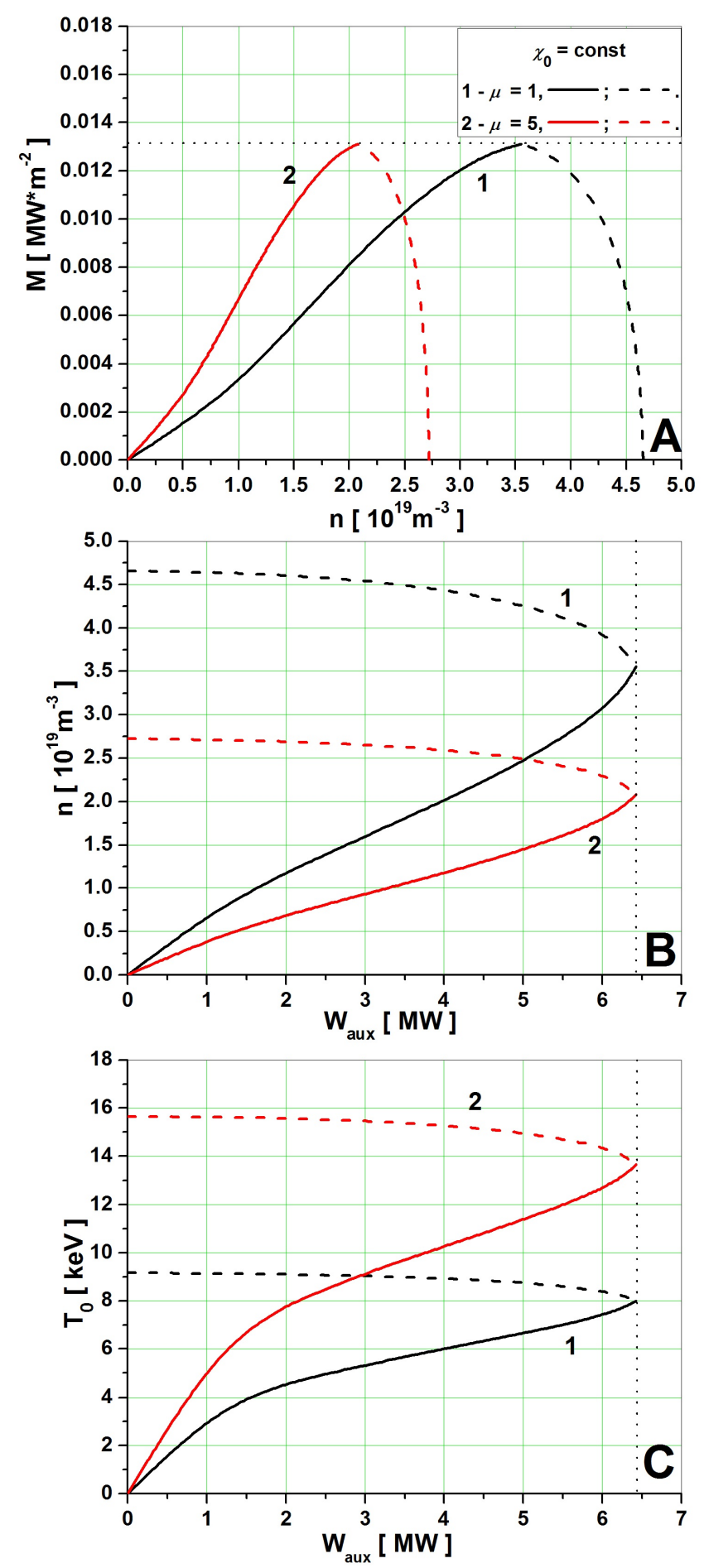

Figure 4. The numerical solution of the equations (19-20) for model $\chi_{0}=$ const. Here impurity is Carbon, $g=4.5 \cdot 10^{-12} \mathrm{eV}^{2} \mathrm{~m}^{3} \mathrm{~s}^{-1}$. Thermal conductivity $\chi_{0}=1 \mathrm{~m}^{2} \mathrm{~s}^{-1}$. Impurity relative concentration $\lambda=0.05$; the minor radius $a=2 \mathrm{~m}$ and the major radius $R=6.2 \mathrm{~m}$.

heating power. Hence the value $Q$ defined as the relation of the fusion power to the auxiliary heating power may be extremely large. The thermal loading at the divertor plate may be small in comparison with ITER case. The critical density and auxiliary heating power related to the transition from the first branch to the second one are found for two models $\kappa_{0}=$ const and $\chi_{0}=$ const. Both of them decrease with the impurity concentration. It is shown that the tokamak-reactor may have the JET like size. 


\section{References}

[1] J.A. Wesson, R.D. Gill, M. Hugon, F.C. Schüller, J.A. Snipes, et al. Disruptions in JET, Nucl. Fusion, Vol.29, No.4, 641-666, 1989.

[2] P.C. de Vries, M.F. Johnson, B. Alper, P. Buratti, T.C. Hender, et al. Survey of disruption causes at JET, Nucl. Fusion, Vol.51, No.5, 053018, 2011.

[3] D.E.T.F. Ashby, M.H. Hughes. A study of the effect of impurity radiation from the peripheral plasma of a tokamak reactor, Nucl. Fusion, Vol.21, No.8, 911-926, 1981.

[4] A. Huber, S. Brezinsek, M. Groth, P.C. de Vries, V. Riccardo, et al. Impact of the ITER-like wall on divertor detachment and on the density limit in the JET tokamak, J. Nucl. Mater., Vol.438, S139-S147, 2013.

[5] M. Greenwald, J.L. Terry, S.M. Wolfe, S. Ejima, M.G. Bell, S.M. Kaye, G.H. Neilson. A new look at density limits in tokamaks, Nucl. Fusion, Vol.28, No.12, 2199-2208, 1988.

[6] V.V. Alikaev, et al. Proc. 17th EPS Conf. on Plasma Phys. and Controll. Fusion, Vol.14B, Part III, p1080, 1990.

[7] A. Stabler, K. McCormick, V. Mertens, E.R. Muller, J. Neuhauser, et al. Density limit investigations on ASDEX, Nucl. Fusion, Vol.32, No.9, 1557-1584, 1992.

[8] T.W. Petrie, A.G. Kellman, M.A. Mahdavi. Plasma density limits during Ohmic L mode and ELMing $\mathrm{H}$ mode operation in DIII-D, Nucl. Fusion, Vol.33, No.6, 929-952, 1993.
[9] Ya.I. Kolesnichenko. The role of alpha particles in tokamak reactors, Nucl. Fusion, Vol.20, No.6, 727-780, 1980.

[10] D.Kh. Morozov, A.A. Mavrin. Heat equilibrium and stability of L-mode in impurity seeded tokamak plasmas, Proc. 41th EPS Conf. on Plasma Phys. Berlin, Germany, P4.033, 2014.

[11] A.M. Messiaen, J. Ongena, B. Unterberg, J. Boedo, G. Fuchs, et al. High confinement and high density with stationary plasma energy and strong edge radiation cooling in the upgraded Torus Experiment for Technology Oriented Research (TEXTOR-94), Phys. Plasmas, Vol.4, No.5, 1690-1698, 1997.

[12] L. Garzotti, P. Belo, G. Corrigan, F. Köchl, J. Lönnroth, et al. Simulations of density profiles, pellet fuelling and density control in ITER, Nucl. Fusion, Vol.52, No.1, 013002, 2012.

[13] D.E. Post, R.V. Jensen, C.B. Tarter, W.H. Grasberger, W.A. Lokke. Steady-state radiative cooling rates for low-density, high-temperature plasmas, Atomic data and Nuclear Data Tables, Vol.20, No.5, 397-439, 1977.

[14] D.Kh. Morozov, E.O. Baronova, I.Yu. Senichenkov. Impurity radiation from a tokamak plasma, Plasma Phys. Rep, Vol.33, No.11, 906-922, 2007. 